\title{
X 波段高温超导滤波器研制
}

周春霞 ${ }^{(1)}$, 夏候海 ${ }^{(1)}$, 左涛 ${ }^{(12)}$, 赵新杰 ${ }^{(1)}$, 方兰 ${ }^{(1)}$ ，阎少林 ${ }^{(1)}$

(1) 南开大学电子科学系, 天津 300071;

(2) 中电集团第 16 研究所, 中电集团低温电子技术研发中心, 合肥 430023

E-mail: zhouchx@mail.nankai.edu.cn

2009-04-08 收稿, 2009-07-28 接受

国家重点基础研究发展计划(编号: 2006CB601006)、国家高技术研究发展计划(编号: 2006AA03Z213)和教育部博士点基金(编号:

200800551009)资助项目

摘要提出了一种综合设计微带带通滤波器的方法, 除了设计微带线的结构, 还综合 考虑了屏蔽盒结构对滤波器技术指标的影响. 利用该方法设计了 X 波段高温超导 (HTS) 微带滤波器. 使用生长于 $0.5 \mathrm{~mm}$ 厚的 $\mathrm{LaAlO}_{3}$ 介质上的 TI-2212 高温超导薄膜, 实现了一个 6 阶带通滤波器, 中心频率为 $9500 \mathrm{MHz}$, 带宽为 $500 \mathrm{MHz}$. 该滤波器具有 极低的带内插入损耗一一于 $0.2 \mathrm{~dB}$, 以及阻带抑制高达 $90 \mathrm{~dB}$ 以上. 在未经调谐的

关键词

低损耗

高抑制

高温超导滤波器

深空探测 情况下, 测试结果与仿真结果基本一致.

基于高温超导(HTS)薄膜具有微波表面电阻小 的特点, 由 HTS 薄膜制作的高温超导滤波器具有插 损小、边带陡峭度高、体积小等优点. 在深空探测网 络中需要极低损耗、极高抑制以及高选择性的 X 波 段滤波器. 由于需要探测非常微弱的信号, 极小插 人损耗、小型化的高温超导滤波器非常适用于深空 探测网络中. 同时为了消除地面发射机频谱信号的 干扰, 需要具有阻带高抑制性能的滤波器. 然而到 目前为止, 大部分工作集中在研究用于通信基站接 收机前端 $\mathrm{S}$ 波段及以下频段的高温超导滤波器 ${ }^{[1-5]}$, 在 $\mathrm{X}$ 波段的卫星通讯应用方面研究较少. 一方面由 于高温超导薄膜的表面电阻正比于频率的二次方, 这使得在 $\mathrm{X}$ 波段高温超导薄膜表面电阻小的优势不 如 $\mathrm{S}$ 波段明显, 不利于实现极低损耗的滤波器. 另一 方面, 在相同介质材料情况下设计 $\mathrm{X}$ 波段和 $\mathrm{S}$ 波段 滤波器时, 由于波长不同, $\mathrm{X}$ 波段高温超导滤波器对 微带线尺寸更敏感. 由加工误差引起微带线宽度的 微小变化, 使得 $\mathrm{X}$ 波段滤波器频响特性偏移仿真结 果要大于 $\mathrm{S}$ 波段滤波器, 这对加工精度提出了更高
的要求.

本文介绍了一种新型的滤波器综合设计方法, 采用尽可能低阶数的滤波器实现阻带上的高抑制, 单一滤波器在阻带上的抑制达到 $90 \mathrm{~dB}$ 以上. 低阶数 的滤波器不仅可以保证低损耗, 还能降低设计以及 制作的复杂性. 同时, 我们选用临界转变温度高, 稳 定性较好, 表面电阻低的高温超导薄膜, 使得该滤 波器满足低损耗的要求.

\section{1 滤波器损耗分析及滤波器设计}

微带带通滤波器的损耗可以表示为 ${ }^{[6]}$

$$
\Delta L_{A 0}^{\prime}=\left(4.343 \sum_{i=1}^{n} \frac{\Omega_{c}}{\mathrm{FBW} Q_{u i}} g_{i}\right) \mathrm{dB},
$$

式中 $\Delta L_{A 0}^{\prime}$ 表示 $n$ 阶带通滤波器在中心频率处的插人 损耗的 $\mathrm{dB}$ 值, $\Omega_{c}$ 为切比雪大低通原形的截止频率, $g_{i}$ 为切比雪大低通原形 $g$ 因子, $\mathrm{FBW}$ 为带通滤波器 的相对带宽, $Q_{u i}$ 为第 $i$ 阶微带谐振器的无载 $Q$ 值, 可以表示为 


$$
\frac{1}{Q_{u i}}=\frac{1}{Q_{c i}}+\frac{1}{Q_{d i}}+\frac{1}{Q_{r i}},
$$

式中 $Q_{c i}$ 表示由导体损耗引起的导体品质因素, $Q_{d i}$ 表示由介质损耗引起的介质品质因素, $Q_{r i}$ 表示由辐 射损耗引起的辐射品质因素.

从(1)式可以知道, 随着带通滤波器阶数的增加, 带通滤波器的损耗也随之增加. 而对于切比雪夫型 带通滤波器, 阶数越大, 陡峭性越好, 带外抑制也越 好. 权衡深空探测中低损耗以及高抑制两个要求, 我们选择波纹系数为 $0.01 \mathrm{~dB}$ 的 6 阶切比雪大型低通 原型。

图 1 为计算得到的理想 6 阶切比雪大带通滤波器 的理论响应曲线. 我们可以看到该滤波器响应在阻 带 $8 \mathrm{GHz}$ 到 $8.2 \mathrm{GHz}$ 的抑制大于 $100 \mathrm{~dB}$. 一般地, 由 HTS 微带滤波器不相邻谐振器之间的交叉耦合带来 的传输零点, 使得 HTS 微带滤波器在通带边缘的陡 峭性很好, 而在远离通带边缘的阻带抑制并不理想. 一般发卡型的 $500 \mathrm{MHz}$ 带宽的 6 阶切比雪夫型 HTS 带通滤波器的带外抑制还不到 $60 \mathrm{~dB}$.

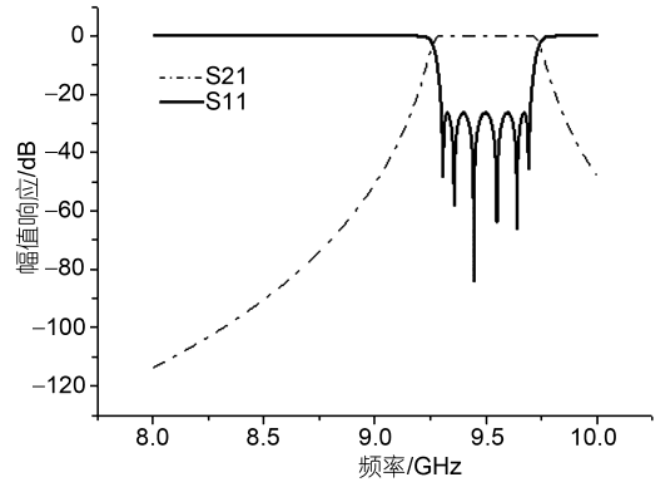

图 1 滤波器理论响应曲线

为了获得阻带高抑制的滤波器, 本文提出了一 种综合设计微带带通滤波器的方法, 除了设计微带 线的结构, 还综合考虑了屏蔽盒结构对滤波器技术 指标的影响. 对于具有相同谐振器结构的微带带通 滤波器, 狭长形的盒子设计有利于提高滤波器的带 外抑制. 由全波仿真软件计算得到盒子宽度与 HTS 滤波器在阻带上 S21 响应值的关系如图 2 所示, 从图 中可以看到, 滤波器盒子宽度由 $24 \mathrm{~mm}$ 减小到 $6 \mathrm{~mm}$ 时, 滤波器阻带抑制由 $40 \mathrm{~dB}$ 增加到 $100 \mathrm{~dB}$. 如果盒 子的宽度继续减小, 滤波器阻带抑制将继续增加.
然而盒子宽度不能选择过窄, 因为微带线离盒子侧 壁越近, 盒子损耗就会越大. 因此, 根据前面分析, 我们选择盒子的宽度为 $6 \mathrm{~mm}$, 此时阻带抑制大于 $100 \mathrm{~dB}$.

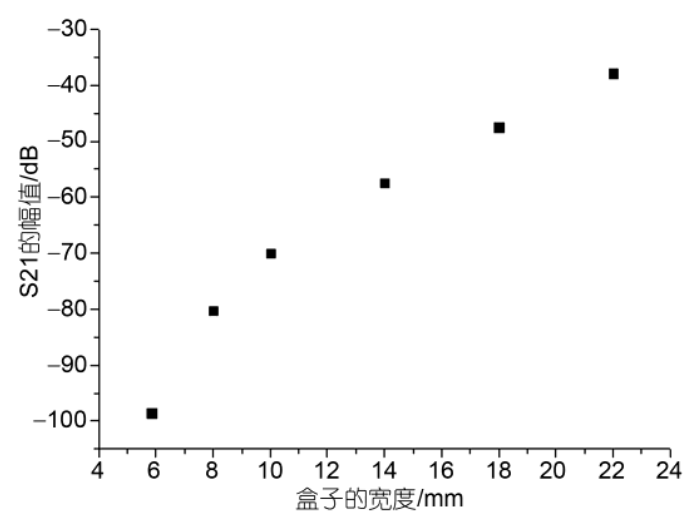

图 2 不同盒子宽度的 HTS 滤波器阻带上 S21 响应值

由波纹系数为 $0.01 \mathrm{~dB}$ 的 6 阶切比雪夫型低通原 型, 可以计算耦合系数矩阵为 ${ }^{[8]}$

$$
\boldsymbol{M}=\left[\begin{array}{cccccc}
0 & 0.970 & 0 & 0 & 0 & 0 \\
0.970 & 0 & 0.660 & 0 & 0 & 0 \\
0 & 0.660 & 0 & 0.621 & 0 & 0 \\
0 & 0 & 0.621 & 0 & 0.660 & 0 \\
0 & 0 & 0 & 0.660 & 0 & 0.970 \\
0 & 0 & 0 & 0 & 0.970 & 0
\end{array}\right],
$$

以及端口耦合系数

$$
R 1=R 2=1.280 .
$$

根据(3)和(4)式, 我们在 $0.5 \mathrm{~mm}$ 厚的 $\mathrm{LaAlO}_{3}$ 介质上设计了中心频率为 $9500 \mathrm{MHz}$ ，带宽为 500 $\mathrm{MHz}$ 的高温超导滤波器, 该 6 阶 HTS 滤波器的版图 如图 3 所示. 该滤波器结构的仿真结果如图 4 和图 5 实线所示, 其中图 4 实线为该滤波器结构在软件 Sonnet's em 下仿真得到的传输特性 S21, 图 5 中实线 为反射特性 S11. 从图中可以看到, 该滤波器的中心 频率为 $9500 \mathrm{MHz}$, 带宽为 $500 \mathrm{MHz}$, 阻带抑制大于 $100 \mathrm{~dB}$.

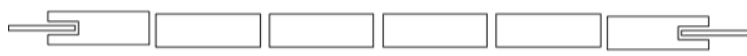

图 3 滤波器版图结构 


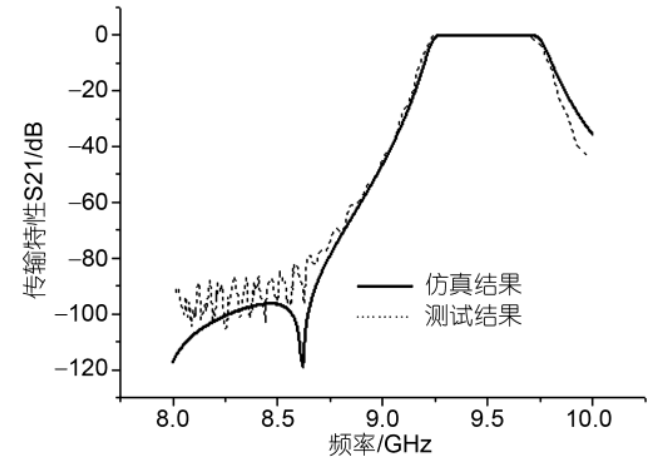

图 4 HTS 滤波器的仿真及测试 S21 响应曲线

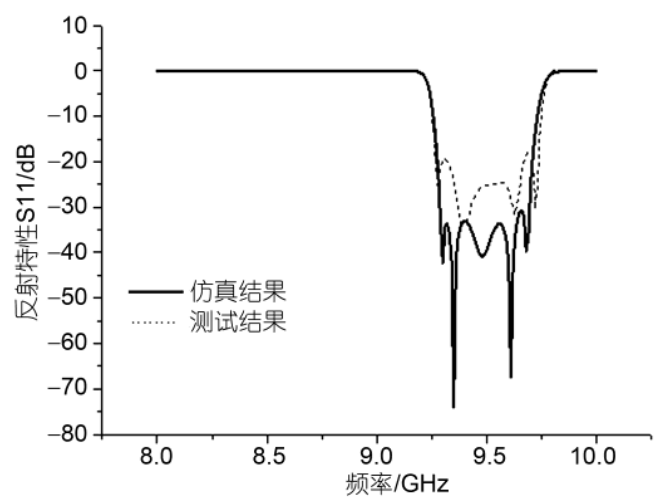

图 5 HTS 滤波器的仿真及测试 S11 响应曲线

\section{2 高温超导薄膜及滤波器制作}

本文研制的滤波器采用的是 $\mathrm{Tl}_{2} \mathrm{Ba}_{2} \mathrm{CaCu}_{2} \mathrm{O}_{8}$ (T1-2212) 高温超导薄膜, 衬底材料采用厚度为 0.5 $\mathrm{mm}$ 的 2 英寸 $(1$ 英寸 $=2.54 \mathrm{~cm}) \mathrm{LaAlO}_{3}(001)$ 基片. T1-2212 超导薄膜是采用 2 步法制作的, 首先用磁控 离子溅射的方法在 $\mathrm{LaAlO}_{3}(001)$ 基片上淀积上双面 Tl-Ba-Ca-Cu-O 非晶先驱薄膜, 然后通过严格的高温 热处理使非晶先驱薄膜转变为 T1-2212 超导薄膜. Tl-2212 超导薄膜的临界转变温度为 $105 \mathrm{~K}$ 左右, 在 $10 \mathrm{GHz}, 77 \mathrm{~K}$ 下的表面电阻低于 $0.5 \mathrm{~m} \Omega$ [9]. 使用这 种 HTS 超导薄膜制作的微带谐振器在 $10 \mathrm{GHz}$ 频率

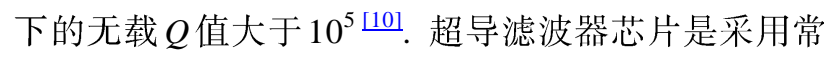
规半导体光刻工艺和离子束刻蚀工艺完成的. 为了 方便超导薄膜微带线与 SMA 微波接头的连接, 在超 导滤波器的输人和输出端口分别蒸镀了金膜.

\section{3 滤波器测试结果与讨论}

本实验使用 Agilent 矢量网络分析仪对滤波器进 行了测试, 同时用制冷机将滤波器冷却到 $77 \mathrm{~K}$ 温度.
测试的滤波器传输特性曲线(S21)如图 4 所示, 实线 是仿真设计的曲线, 虚线是实际测试结果. 可见滤 波器实际测试结果与仿真设计的曲线吻合得比较好, 在未经调谐的情况下, 测试结果显示中心频率和带 宽与设计基本一致, 阻带抑制好于 $90 \mathrm{~dB}$. 图 6 为传 输特性 S21 在通带内的放大曲线图, 由图可以看出 在通带内的插人损耗好于 $0.2 \mathrm{~dB}$. 在对网络分析仪 进行校准时, 我们选择与高温超导滤波器相连的刚 性同轴连接器作为校准端口, 因此测得的插入损耗 只包括滤波器本身损耗及其两个 SMA 接头损耗. 图 5 给出了滤波器的仿真及测试的回波损耗(S11)响应 曲线. 实线是仿真设计的曲线, 虚线是实际测试结 果. 实测带内回波损耗好于 $18 \mathrm{~dB}$, 略大于仿真结果. 这是由于在实际加工过程中, 由于焊接原因造成 SMA 接头与滤波器 $50 \Omega$ 馈线处不完全匹配以及微 带线光刻的误差造成的.

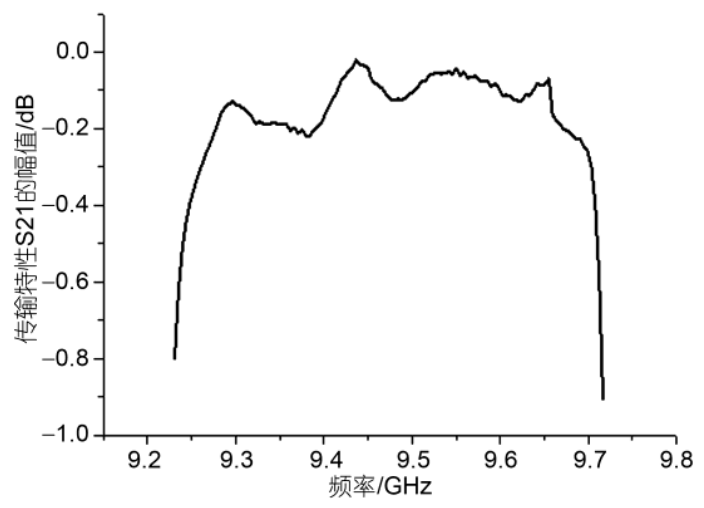

图 $6 \mathrm{S21}$ 通带内的放大曲线图

\section{4 结论}

本文讨论了 X 波段高温超导滤波器的高性能指 标, 通过理论分析及电磁仿真设计, 总结出一种新 颖的微带带通滤波器的综合设计方法, 结合深空探 测网络中极低损耗、阻带高抑制性能的技术要求, 我 们选用生长在 $\mathrm{LaAlO}_{3}$ 衬底上 Tl-2212 高温超导薄膜, 通过相关的综合设计, 实现了一个可用于深空探测 网络中的具有极低插人损耗, 阻带高抑制性能的 HTS 带通滤波器. 在未经调谐的情况下, 滤波器的 带内插人损耗好于 $0.2 \mathrm{~dB}$, 阻带抑制好于 $90 \mathrm{~dB}$. 此 滤波器满足深空探测网络中的技术要求. 


\section{参考文献}

1 Zuo T, Fang L, Wu Z, et al. A self-equalized HTS filter for future mobile. Physica C, 2007, 459: 1-4 $\underline{\text { [doi] }}$

2 Ning J S, Yang K, Luo Z X, et al. Design of high temperature superconducting contiguous-band diplexer. Chinese Sci Bull, 2009, 54: $1279-1281$ [doi]

3 Xia H H, Zhou C X, Zuo T, et al. Development of high temperature superconducting filters operating at temperatures above $90 \mathrm{~K}$. Chinese Sci Bull, doi: 10.1007/s11434-009-0347-4

4 Hong J, Lancaster M J, Jedamzik D, et al. On the development of superconducting microstrip filters for mobilecommunications applications. IEEE Trans Microw Theory Tech, 1999, 47: 1656-1663[doi]

5 Zhang X, Meng Q, Li F, et al. A 24-pole high Tc superconducting filter for mobile communication applications. Supercond Sci Technol, 2006, 19: 394-397[ [doi]

6 Hong J S, Lancaster M J. 2001 Microstrip Filters for RF/Microwave Application. New York: Wiley, 2001

7 刘娟秀, 羊恺, 罗正祥, 等. 星载高温超导滤波器研制。通信学报, 2006, 27: 105-109

8 Richard J C. General coupling matrix synthesis methods. IEEE Trans Microw Theory Tech, 1999, 47: 433-442 [doi]

9 Yan S, Fang L, He M, et al. $\mathrm{Tl}_{2} \mathrm{Ba}_{2} \mathrm{CaCu}_{2} \mathrm{O}_{8}$ thin films on 2in. $\mathrm{LaAlO}_{3}(001)$ substrates. Cryogenics, $2005,45: 41-44$ [doi]

10 Zhang X, Yan S, Ji L, et al. Microwave surface resistance measurement of hts films using dielectric resonators. Physica C, 2006, 449: 96-99[ [doi] 